\title{
تطرير المذكّرة لشرح نظم العمريطي بخريطة المفاهيم \\ بمدرسة منبع الصالحين المثتوسطة جرسيك
}

Ida Fauziyatun Nisa

IAI Sunan Giri Bojonegoro, Indonesia

Email: idafauzi91@gmail.com

\begin{abstract}
Qowaid have an important role in the understanding of the Arabic language, As for the difficulties faced The female students MTs Mambaus Sholihin is difficult to understand the book Nahwu (Syarah Nadhom Imrithy) The book requires a development in form, so it fits with the desire of the students of today. The development eg by using electronic media are being developed today. therefore, researchers developed the book so that the students can understand them well. His research is teaching materials developed with the following steps (1) preparing the materials (2) preparing media for development (3) the implementation of the development (4) Evaluation of the questionnaire Validator for advice and criticism and test students to get the data about the effectiveness of the implementation of the Product. from the results of the questionnaire validator that the data obtained Handout to Sarah Nadhom Imrithy with Mindmapping can be used in the learning process with minor repairs. And from the results of data analysis tests showed that the handouts for Sarah Nadhom Imrithy with Mindmapping Effective to improve the material kefahaman nahwu. And from the student questionnaire responses obtained from the data $89 \%$ indicate the criteria nice ".
\end{abstract}

Kata kunci : Pengembangan, Handuot, Syarah Nadhom Imrithy, Mind Mapping

مقدمة

قواعد اللغة العربية له دور مهم في فهم اللغة العربية، عندما تعتبر القواعد صعبة وخخيفة يمكن أن يؤدي الطلبة بعيدا عن اللغة العربية وانخفض دوافعهم لتعلّم اللغة العربية. من القواعد العربية النحو، قال مصطفى الغلاييني، النحو هو دراسة لغوية تركز في تركيب الكلمات كعناصر الجملة من الكالام، فلكل لغة دراسة نحوية التي تقصد بها حفظ 
المتكلّم من الخطاء في تأدية المعنى لإيصاله إلى ذهن السامع من أغراض. فالنحو نتيجة عمل عقلي مستمر في فهم تركيب الجملة في لغة معينة، وفي شأنه أن ينمو اللغة

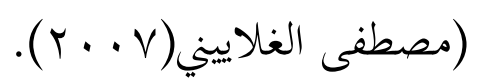

قال إبن جني أن النحو هو "إنتحاء سمت كلام العرب، في تصرفه من إعراب وغيره، كالثنية، والجمع، والتحقير، والتكسير، والإضافة، والنسب، والتركيب، وغير إنهاء

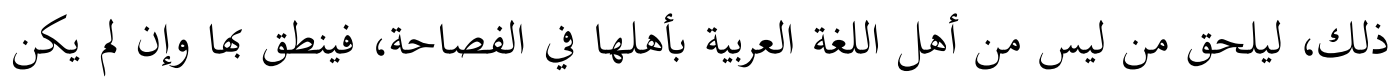

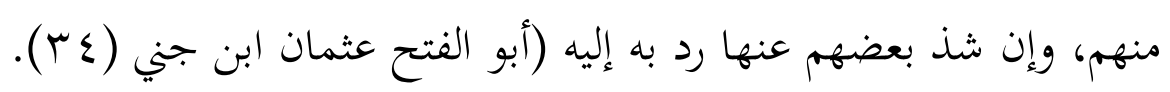
إن الغرض من تدريس النحو هو تكوين الملكة اللسانية الصحيحة، لاحفظ القواعد المجردة، فالعرب الأول الذي أخذت عنه اللغة، لم يكن يدري النحو ما الحوين الحال وما

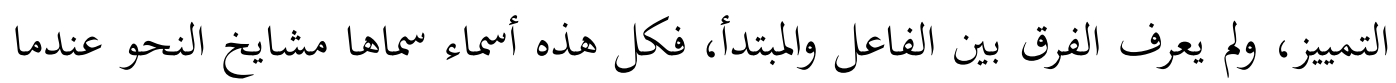

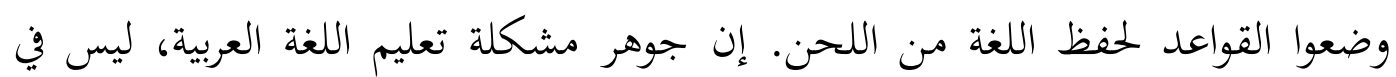
اللغة ذاتما، وإنما في كوننا نتعلم اللغة قواعد جافة، وإجراءات تلقينية، وقوالب صماء،

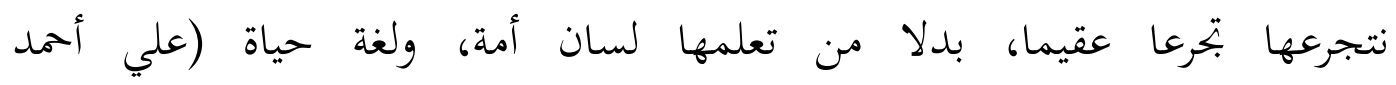

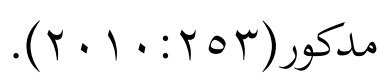
يدرّس النحو كمادة مستقلة من العلوم العربية في كثير من المؤسسات الإسلامية بإندونيسيا من المستوى الإبتدائي إلى الجامعي. ولكن للأسف يعد معظم الطلبة الذين يدرسون النحو أنه مادة مخيفة، بحيث يجتنبه الطلبة. المدرسة المتوسطة منبع الصالحين إحدى المدارس التي يتعلم فيها علم القواعد

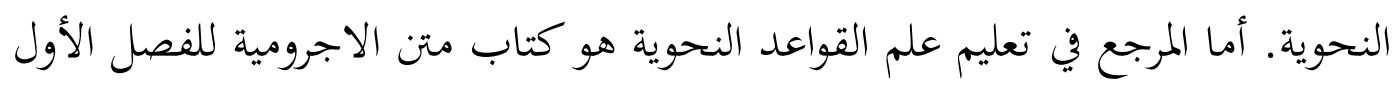
وشرح نظم العمريطي للفصل الثاني والثالث. سئلت الباحثة معلمات القواعد النحوية عن أهداف تعليم القواعد النحوية وطريق تعليمها ودوافع الطالبات لتعلّمها وكفاءة الطالبات في فهمها، قالت المعلمات أن أهداف تعليمها (1) تستطيع الطالبات فهم اللغة العربية. (r) تستطيع الطالبات تكلم باللغة العربية الفصحى. (r) تستطيع الطالبات قراءة كتب 
التراث. ومن طريق تعليمها طريقة التقليدية وإعطاء المثال والمناقسة والتطبيق بقراءة كتب التراث. وتنعقد تلك المدرسة إحتفال ختم العمريطي في فاية الفصل الثالث لارتفاع دوافع الطالبات، عقدت لجنة الإحتفال الإنتقاء ثلاث مراحل، يعني: (1) حفظ النظم

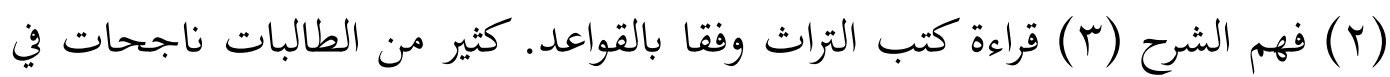
المرحلة الأولى بل غير ناجحات في المرحلة الثاني. يشير هذا أن فهم شرح نظم العمريطي صعب عند الطالبات.

تجعل صعوبة الطالبات لفهم كتاب شرح نظم العمريطي، هذا الكتاب يحتاج الى التجديد في صورته حتى يكون اكثر مناسبة لرغبة طلبة العلوم اليوم باستخدام التطورات المتوفرة اليوم، لذالك طورت الباحثة ذلك الكتاب هدفا لمساعدة الطالبات فهم ذلك بكريه

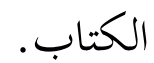

من المشكلات السابقة إجتهدت الباحثة تطوير المذكّرة (Handout) بخريطة المفاهيم لشرح نظم العمريطي كي يكون ذلك الكتاب واضحا وسهول الفهم عند الطالبات. يتضمن في المذكّرة (Handout) خريطة المفاهيم لشرح نظم العمريطي والبيان والتدريبات. ظهر خريطة المفاهيم توني بوزان (Tony buzan) يعني تقنيات التصوير في

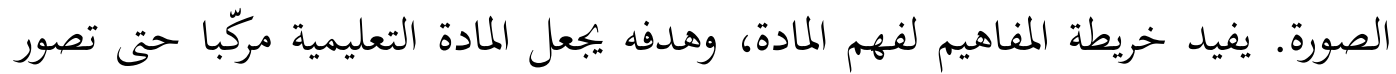
في ذهن الطلبة تعزيز الذاكرة و يذكر الدروس التي قد تُعِّمِت وتساعد خريطة المفاهيم حلّ مشكلات التعليم إبتكاريا ( Toni Buzan (2012:4). أ. أ. مفهوم المذكّرة(Handout)

المذكّرة هي كل شيئ الذي يعطي الى الطالبات عند عملية التعليم. والتعريف الآخر هي المواد المكتوبة المستعيدة للطالبات لتحصيب معلوماتن .

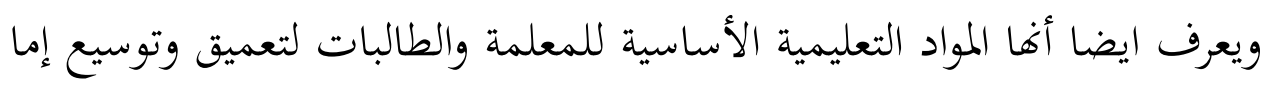
في عملية التعليم او المعمل او الميدان بواسطة الدراسة او المصادر الواجبة او المراجع. 
ب.وظائف المذكّرة (Handout) ب (ب)

رأي ستيفن و فتر بلستد (Staffen dan Peter Ballstaedt) من وظائف المذكّرة

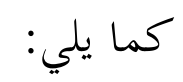

$$
\begin{aligned}
& \text { 1. يساعد الطالبات } \\
& \text { r r r كقرين شرح المعلم } \\
& \text { r. (بمراجع للطالبات } \\
& \text { ـ. دعا للطالبات كي تكون نشيطة في التعلم. } \\
& \text { ه. ذكر المادة الأساسية التي قد تعلّمت } \\
& \text { 7. } \\
& \text { V } \\
& \text { ج. غرض بناء المذكّرة (Handout) } \\
& \text { في أنشطة التعلم لنشرة الأهداف، كما يلي: }
\end{aligned}
$$

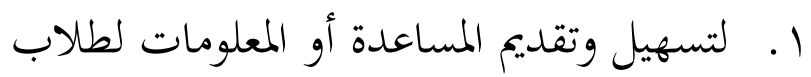

$$
\begin{aligned}
& \text { r. لإثراء معرفة الطلاب } \\
& \text { r. د. دعم المواد التعليمية الأخرى أو تعليل المعلمين }
\end{aligned}
$$

د. خصائص المذكّرة(Handout)

$$
\text { رأى سودجاتي (Sudjati) أن المذكّرة لما خصائص، يعني: }
$$

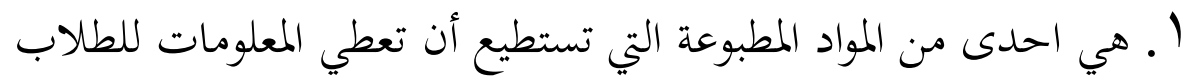

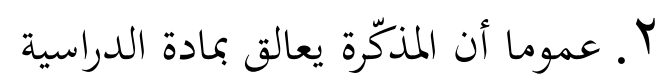

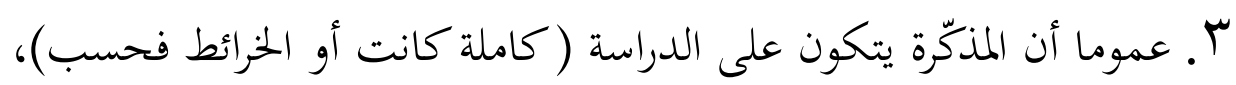

$$
\text { او الجداول أو المادة الزائدة. }
$$

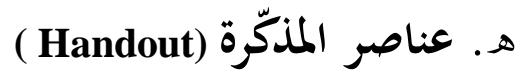

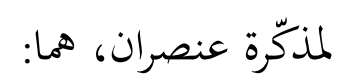




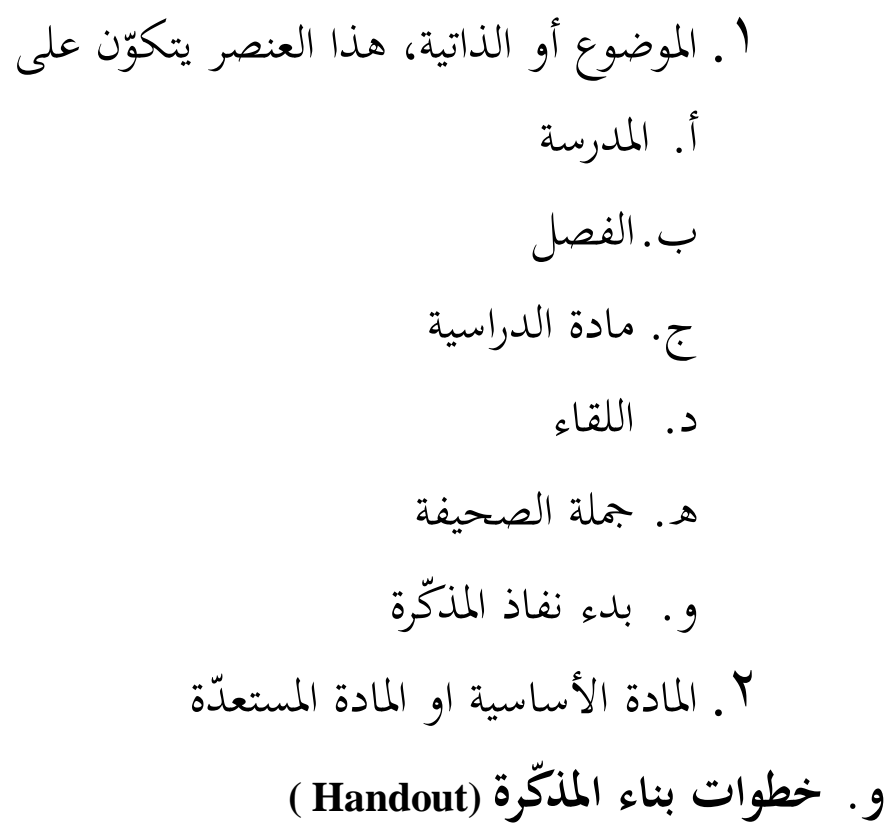

تتم المذكرة بدليل على الكفاءة الأساسية التي يجب تحقيقها الطلاب. فلذلك يجب بناء المذكرة على أساسي المنهج. أما الخطوات كما يلي:

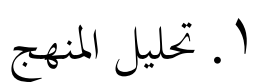
Y. تحقيق موضوع المذكرة ويناسب على الكفاءة الأساسية والمادة التعليمية المتحققة "ا. جمع المراجع لدليل بنائها ع. لا يكتب المذكرة بكلمة طويلة ○. تقويم الكتابة بقراءة مراة ثانية أو طلب المداخلات من الخبير 7. تحسين المذكرة بحسب النقصان الموجودة(2014:82) Andi Prastowo.). ز. مفهوم خريطة المفاهيم

عبارة عن رسم تخطيطي تترتب فيها مفاهيم المادة الدراسية في تسلسل هرمي، وبطريقة البعد الراسي، حيث تترابط المفاهيم بحيث تتدرج من المفاهيم الأكبر شمولية (عمومية) إلى الأقل خصوصية (فرعية ) إلى المفاهيم الأقل شثملية والأكثر خصوصية، حيث تحتل المفاهيم الأكثر شثمولية قمة خريطة المفاهيم، وكلما ابتهنا إلى أسفل تقابلنا 
المفاهيم الأقل شثملية. وتعرف بأغها: عبارة عن رسوم تخطيطية تدل على العلاقة بين المفاهيم، وهي تحاول أن تعكس التنظيم المفاهيمي لفرع من فروع المعرف، وهذه الرسوم يمكن أن تكون ذات بعد واحد أو بعدين (عزواسماعيل عفانة). ح. فوائد خريطة المفاهيم الخرائط المفاهيمية لها فوائد الكثيرة، منهن: ا ـ تساعدنا للتخطيط و الإتصال Y ب التعلنا زيادة الإبتكار

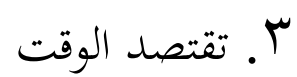
ك. تحلّ المشاكل 0. تركيز الإهتمام Y I تصميم وتشريح الفكرة

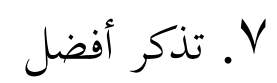
^. التعلم أسرع و أكثر كفاءة 9 - ونى الصورة كاملة. ط. خطوات بناء خريطة المفاهيم

بنيت الباحثة الخرائط المفاهيمية بسبع خطوات، يعني:

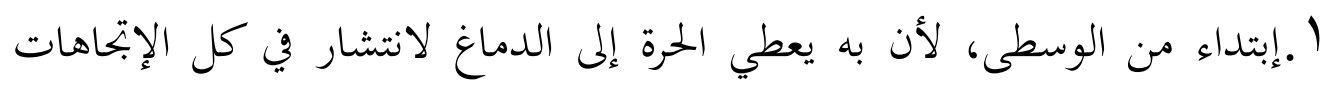
ولتعبير أنفسه.

Y.إعطاء الصورة للفكرة الرئيسية لأهما معانى متعددة وساعدت لاستخدام الهادف

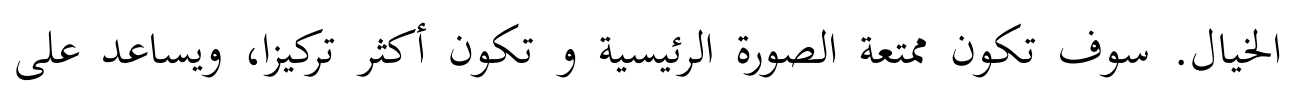

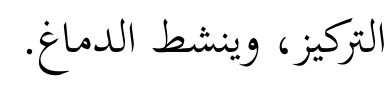
r.إستخدام اللون لأنه متساويا بالصورة في الإمتاع. يجعل اللون الخزائط المفاهيمية حيوية. 
ع.ربط الفروع الرئيسي للصورة المركزية وربط الفروع مستوى الثاني والثالث إلى ع مأل

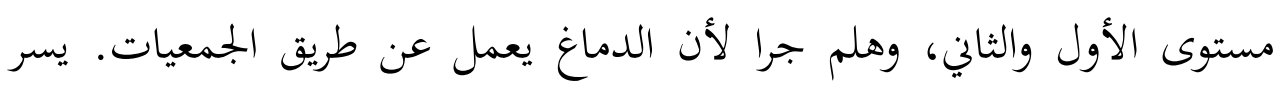

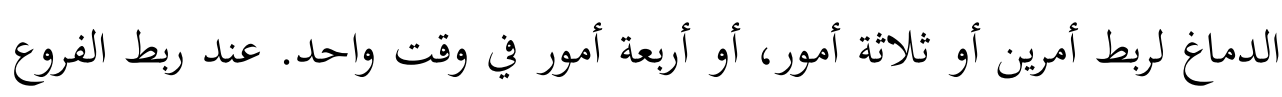
سيكون الفرع أسهل للفهم وتذكر.

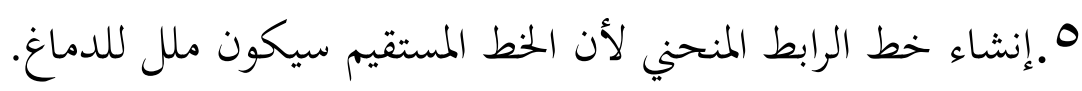
7 7. إستخدام الكلمة الرئيسية لكل خط، لأهفا تزيد القوة والمرونة لخريطة المفاهيم. استخدام الصورة لكل الصورة معانى متعددة ( Toni Buzan (2012:4). ي. كتاب نظم العمريطي ومؤلفه

اسم مؤلف كتاب نظم العمريطي يمي بن موسى بن رمضان بن عميرة العمريطي الشافعي شرف الدين فقيه أصولي ناظم توفي في حدود سنة وموهـ من قرية عمريط مركز

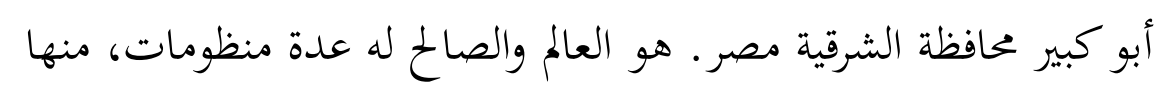

ا (يف بجال الفقه • التفسير في نظم التحرير " هاية التدريب في نظم الغاية والتقريب • تسهيل الترقات في نظم الورقات

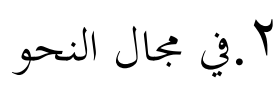

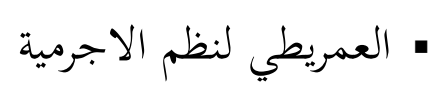

هو نظم من كتاب الاجرمية لشيخ ابو داود الصنهجي. هذا الكتاب مختصرة

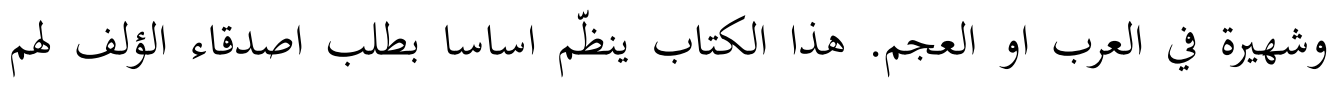

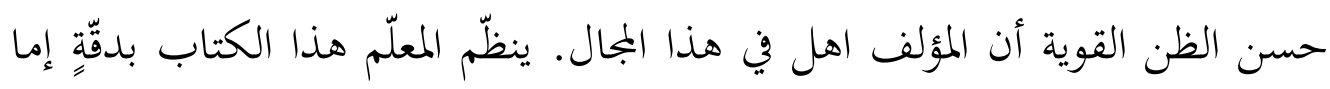

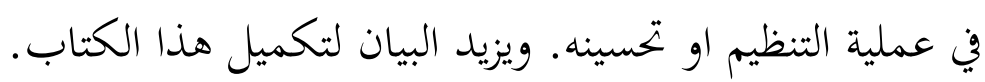


كتاب نظم العمريطي يتضمن على ثلاثة وثلاثون بابا يعني : (1) المقدمة،

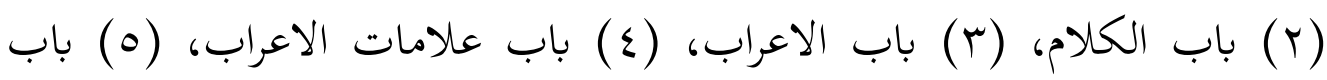

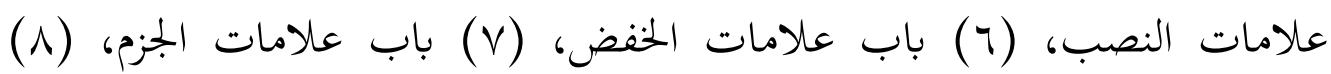

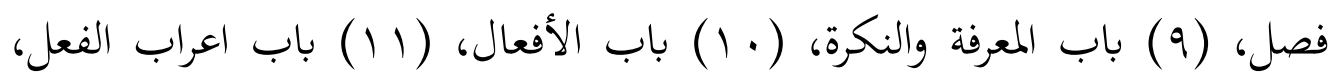

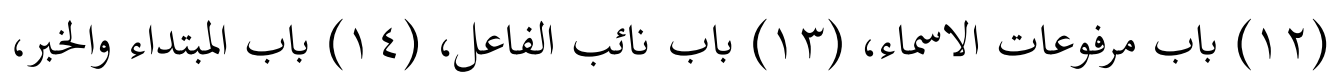

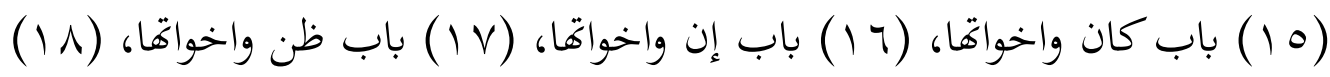

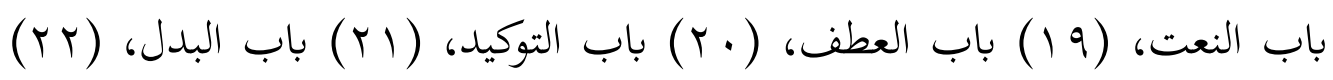

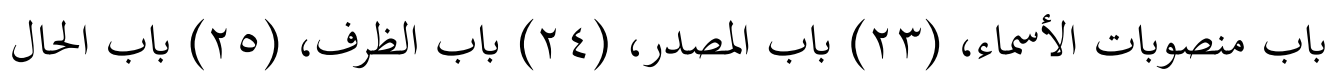

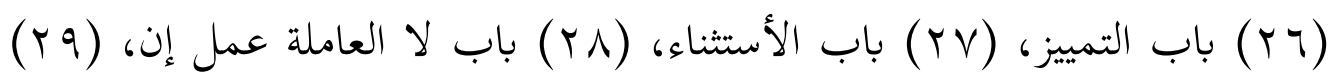

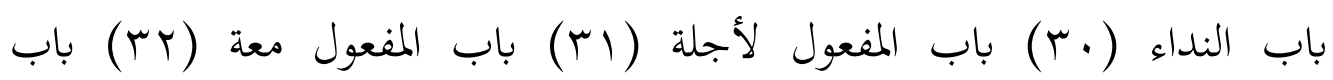
محفوضات الاسماء (rr) باب الإضافة. فيه النظم والترجمة ثم تتطوّر الباحثة هذا

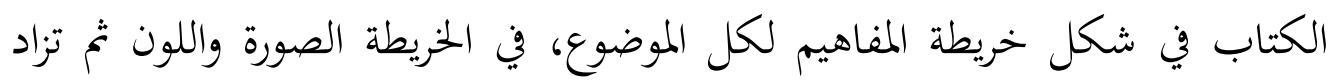
بالبيان الإضافي الذي نقل من كتاب ألفية ابن مالك وفي الاخير تقديم التدريبات لقراءة كتب التراث.

ك. مدخل البحث ومنهجه

Research \& ) في هذا البحث استخدمت الباحثة منهج البحث والتطوير

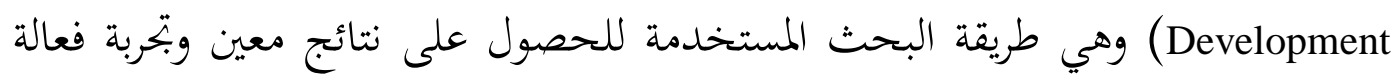

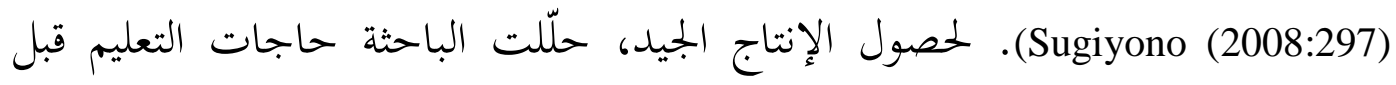
عملية التطوير. ويستند هذا البحث إلى المدخل الكيفي والكمي . أما النموذج الذي إنداجي تستخدمه الباحثة في إجراء هذا البحث هو نموذج Borg \& Gall الذي قد شرحه سوكيونو ( Sugiyono ) في كتابه ل ل. إجراءات البحث والتطوير 
في تطوير شرح نظم العمريطي ستستخدم الباحثة نموذج Borg \& Gall الذي قد شرحه سوكيونو ( Sugiyono ) في كتابه، وهو يشتمل على إجراءات التالية : (1) تحليل الحاجات ومشكاتلات. (r) جمع المعلومات. (r) تصميم الإنتاج. (ع) تصديق الإنتاج (0) تحسين الإنتاج. (7) بتربة صلاحية (V) تحسين الانتاج (م) بتربة الميدانية. (9) (9) تحسين الإنتاج. ( • (1) النهائي.

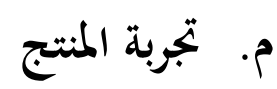

تمدف هذه التجربة كما ذكر الباحثة فيما سبق، لرفع فعالية الكتاب التعليمي، وأيضا لحصول الاستجابات و الاقتراحات والتقويمات عن صالحية الكتاب التعليمي في مادة القواعد النحوية في مدرسة منبع الصالحين المتوسطة. وبالتالى تعرض الباحثة: تصميم التجربة

إن بتربة هذا الكتاب التعليمي تجري على ثلاث مراحل،وهي كما يلي: أ. المرحلة الأولى هي النظرة والإقتراح من خبيرين منهما: خبير مادة تعليم النحو وخبير في تصميم المادة التعليمية. جعلتها الباحثة أساسا لإصلاح وتصحيح

$$
\text { المذكّرة (Handout) لشرح نظم العمريطي. }
$$

ب. والمرحلة الثانية تجربة بجموعة صغيرة من الطالبات، جعلتها الباحثة لتصحيح

$$
\text { الأخطاء من المذكّرة (Handout) لشرح نظم العمريطي }
$$

ج. والمرحلة الثالثة بتربة ميدانية، جعلتها الباحثة أساسا لمعرفة صلاحية وتناسب المادة التعليمية بالطالبات في الفصل الثاني بمدرسة منبع الصالحين المتوسطة

$$
\text { سوبتجي مانيار جرسيك. }
$$

$$
\text { أفراد التجربة }
$$

إن القيام بالتجربة يحتاج إلى أفراد التجربة، وفيما يلي أفراد التجربة: أ. النظرة والإقتراح من الخبيرين 
إن الفاعل في هذه النظرة والإقتراح هو خبير مادة تعليم النحو وخبير في

$$
\text { تصميم المادة التعليمية. }
$$

من هذه التجربة ستحصل الباحثة البيانات يعني التعليقات والتوصيات عن

$$
\begin{aligned}
& \text { الإنتاج من خلال مادة المضمون (النحو) و تصميم الإنتاج } \\
& \text { ب. التجربة الفردية }
\end{aligned}
$$

إن الفاعل في هذه تجربة بجموعة صغيرة هو اربع الطالبات بممدرسة منبع

$$
\text { الصالحين المتوسطة سوتجي مانيار جرسيك. }
$$

والفاعل في هذه التجربة الميدانية هو خمس وتسعون طالبات، تنقسمهن الباحثة على الفرقتين. الفرقة الأولى فرقة ضابطة فيها ثمانية واربعون طالبات، والفرقة الثاني فرقة تجريبية فيها سبعة واربعون طالبات كلهن من الفصل الثاني بمدرسة منبع الصالحين المتوسطة سوتجي مانيار جرسيك. من هذه التجربة ستحصل الباحثة البيانات عن فعالية الإنتاج البيانات والمعلومات

$$
\begin{aligned}
& \text { أما البيانات والمعلومات في هذا البحث فيما يلي: } \\
& \text { أ. أبيانات الكيفية } \\
& \text { هذه البيانات تشتمل على: }
\end{aligned}
$$

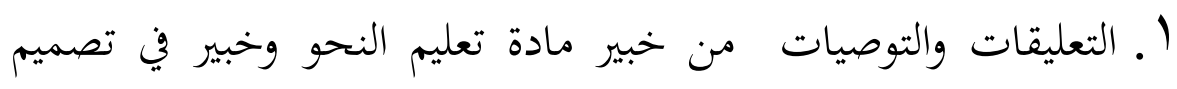

المادة التعليمية.

ץ. نتائج المقابلة من معلمات تعليم القواعد النحوية لدى طالبات في مدرسة منبع الصالحين المتوسطة سوبتي مانيار جرسيك وكيف رأيهن

$$
\text { ". نتائج المقابلة عن عملية التعليم والتعلّم كتم العمريط في ضوء الخرائط المفاهيمية. }
$$




$$
\begin{aligned}
& \text { ب. البيانات الكمية } \\
& \text { تشتمل هذه البيانات على: } \\
& \text { ' ـ نتائج التحكيم عن نوعية الكتاب التعليمي المطورة }
\end{aligned}
$$

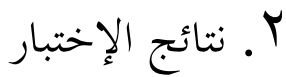

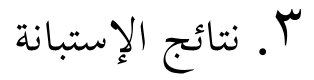

أسلوب جمع البيانات

تستخدم الباحثة أدوات البحث اللآتية:

$$
\text { أ. أ. ماحظة }
$$

لاحظت الباحثة كتاب تعليم القواعد المستعمل في المدرسة المتوسطة منبع

الصالحين، ولاحضت كيف عملية تعليمه و كيف فعاليته. وشكل الملاحظة في هذا البحث هو المالاحظة غير محدودة لأن مركز البحث ستتطور مادام جار عملية البحث(Sugiyono (2008:146). ب. المقابلة

قابلت الباحثة بمعلمات علم النحو عن الأهداف الأساسية من تعليم علم

النحو في مدرسة منبع الصالحين المتوسطة ، ومن عملية تعليم علم النحو فيه حول المنهج المستخدمة والخطة الدراسة والمواد التعليمية والكتب المستخدمة وطريقة المستعملة والوسائل التعليمية وطريقة التقويم والمشكالت التي يواجهها معلمة تعليم علم النحو في العملية التعليمية. وقابلت الباحثة بالخبراء لنيل التعليقات في بحال مواد تعليم علم النحو وتصميم الانتاج ولنيل الإرشادات لتحسين هذا المواد المعدة في صورة أحسن حتى يصل إلى فعالية تطبيقه في عملية تعليم علم النحو في مدرسة منبع الصالحين المتوسطة سوبجي مانيار جرسيك. ج. الإستبانة 
تستخدم الباحثة الاستبانة المقيدة والاستبانة المفتوحة. الاستبانة المقيدة هي الاستبانة التي تطلب من المستجيب أن يختار إحدى الاجابات المكتوبة في تلك الاستبانة دون غيره. وأما الاستبانات المفتوحة هي الاستبانة التي تعطي المستجيب الفرصة ليجيب على كل الأسئلة فيها بحرية حسب رأيه وبالجمل

$$
\text { أوالعبارات التي من عنده. }
$$

تستخدم الباحثة الاستبانة المقيدة لجمع البيانات عن حاجات الطالبات في تعليم علم النحو ورغبتهم في مواقف وموضوعات مواد تعليم علم النحو. وتستخدم الباحثة الاستبانة المفتوحة لمعرفة صلاح المواد المعدة للتطبيق من الخبراء والمعلمات والطالبات في مدرسة منبع الصالحين المتوسطة سوتجي مانيار جرسيك، ولمعرفة ارآهن حول ذلك المواد.

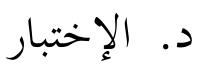

تقديم الباحثة إختبارين في هذا البحث، هما الإختبار القبلي والإختبار البعدي. الإختبار القبلي هو الإختبار الذي يعطي قبل البرنامج الدراسي، أما الاختبار البعدي هو الاختبار الذي يعطي بعد البرنامج الدراسي (محمد علي الخالي(10:0 (19910).

ستحصل من هذا الإختبار البيانات عن فعالية الإنتاج من تطوير كتاب شرح نظم العمريطي، منها: تحليل البيانات

$$
\text { | (تحليل البيانات الكيفية }
$$

استخدمت الباحثة نموذج ملس و هيبرمين (Miles and Huberman) لتحليل نتائج المقابلة المفتوحة مع معلمات شرح نظم العمريطي لمعرفة احتياجاهن إلى المواد المطورة من شرح نظم العمريطي على ضوء خريطة المفاهيم وتحليل نتائج ملاحظة الباحثة في مجال عملية التعليم والتعلّم، كذلك صنك 
تحليل نتائج الاستبانة المفتوحة التي تحتوي على تعليقات خبيري المواد التعليمية وتصميم المواد التعليمية عند تصديق المواد التعليمية المطورة. ويجرى تحليل هذا البيانات على ثلاث مراحل (Sugiyono (2008:246): ا ـ المرحلة الأولى : تقصير البيانات (Data Reduction)

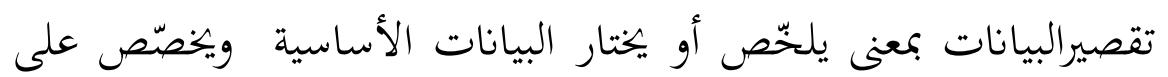

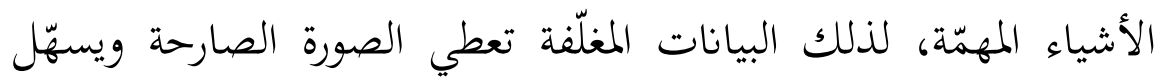
الباحثة لجمع البيانات الآتية. Y. المرحلة الثانية : تقديم البيانات (Data Display) تستطيع تقديم البيانات في شكل الخلاصة أو رسم بياني وعلاقة بين

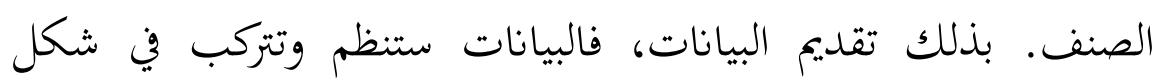

$$
\text { بـ العلاقة، حتى يسهل فهمها. }
$$

الخطوة الثالثة في تحليل البيانات الكيفية هي الاستنتاج والتحقق . الاستنتاجات الأولية المقدمة لا تزال مؤقتة، وسوف تتغير إذا لا يوجد

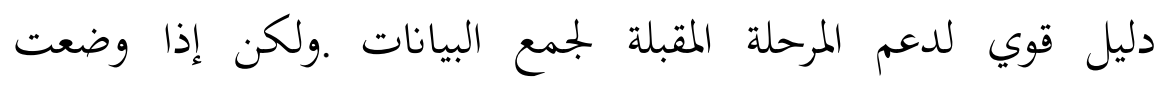

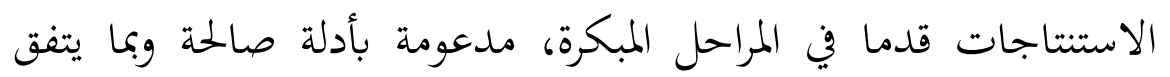
عندما عادت الباحثة إلى الميدان لجمع البيانات، وضعت الاستنتاجات

$$
\begin{aligned}
& \text { المتقدمة استنتاج مصداقية } \\
& \text { r.تحليل البيانات الكمية } \\
& \text { أ. أسلوب تحليل البيانات من الإستبانة }
\end{aligned}
$$

قامت الباحثة بتحليل البيانات المتوفرة من خلال إستبانة التحكيم البناله

من الخبراء ومعلمات القواعد النحوية والطالبات في الفصل الثاني بمدرسة 
منبع الصالحين المتوسطة لتصوير آرائهم عن تطوير كتاب شرح نظم

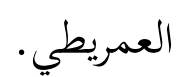

إستخدمت الباحثة في هذه الإستبانة مقاييس رتيع سكيل، وهي أسلوب لقياس السلوكيات والتفضيلات مستعمل في الإختبار النفسية. وفي هذه المقياس أن الطالبات لايلزمن لإختيار الأسئلة السلبية ولكن أيضا إختيار الأسئلة الإيجابية (2005:159) (Zainal Arifin).

$$
\text { ب.أسلوب تحليل البيانات من الإختبار }
$$

إستخدمت الباحثة في تحليل البيانات من نتيجة الإختبار القبلي

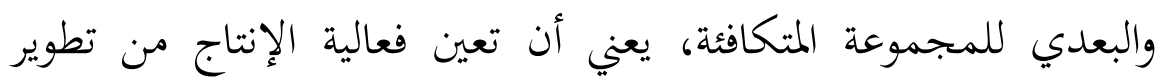
كتاب شرح نظم العمريطي وعدمها تقاس حسب نتيجة الطلاب في

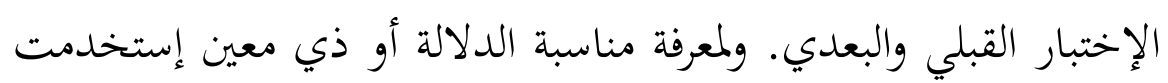

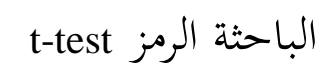

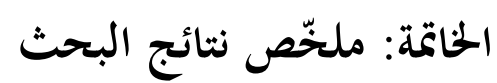

بعد أن قامت الباحثة بعرض البيانات وتحليلها ومناقشتها في الفصل السابق،

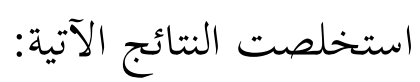

ا ـ طوّرت الباحثة المذكرة لشرح نظم العمريطي بخريطة المفاهيم بمدرسة منبع الصالحين المتوسطة جرسيك. هذه المذكرة تصمم بالخطوات التالية (1) اعداد

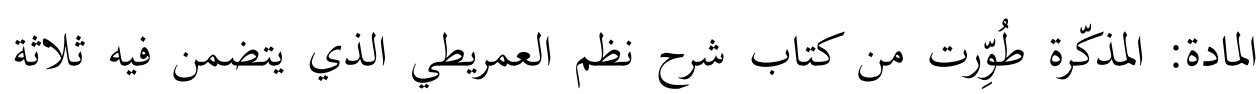
وثلاثون بابا. (Y) استخدمت الباحثة برماجيات Edraw Mind Map لتطوير

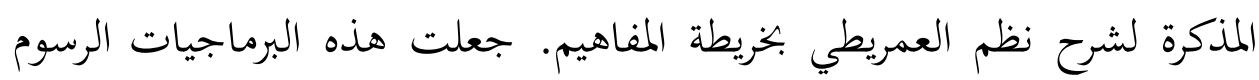
البيانية وخريطة المفاهيم يمكن استخدامها بسهولة جدا وكانت النتائج جيدة جدا.(r) تنفيذ التطوير: هذه المذكرة تتضمن من ثلاثة وثلاثين بابا مناسبا

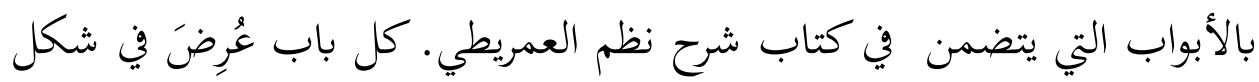


خريطة المفاهيم ويُزاد فيها البيان الإضافي الذي نُقِل من كتاب ألفية ابن مالك واختُتمت بالتدريبات لقراءة كتب التراث.صفة هذا الإنتاج مطبوعة، حجم ورقته

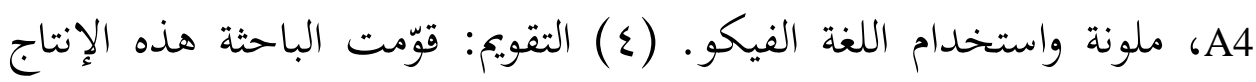
بتقديم الإستبانة للخبيران. خبير في تصميم المواد وخبير في محتوى المواد التعليمية. هذه التقويم لنيل المداخلات والإقتراحات والإرشادات البناءة لتكون المواد التعليمية المطورة على الصورة المرجوة.وقوّمت الباحثة هذه الإنتاج بتقديم الاختبار القبلي والبعدي للفصل الضابط والفصل التجريبي. بهذه الاختبار حصلت الباحثة البيانات عن فعالية الأنتاج.

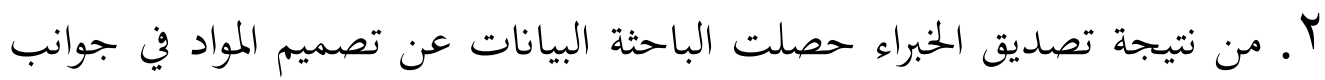

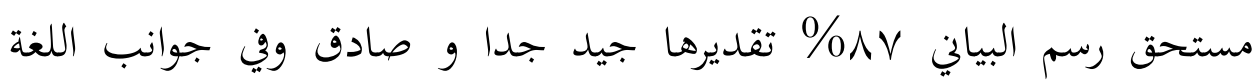

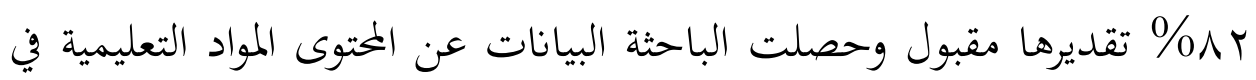

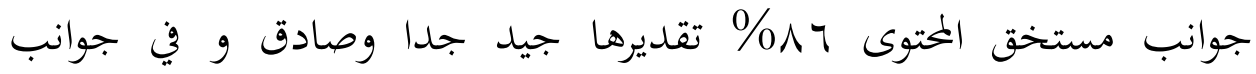

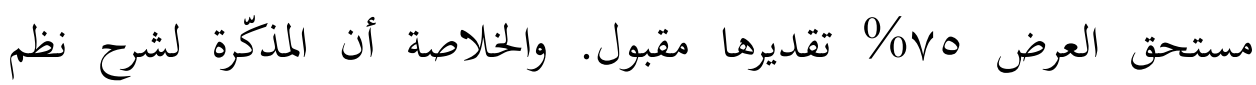
العمريطي بخريطة المفاهيم يمكن استخدامها في التعليم بالتصحيح البسيط. ؟ـ إن المواد المطوّرة (المذكّرة) على الوجه العام جيدة ولها فعالية في ترقية فهم مادة النحو لدى الطالبات في الفصل الثاني بمدرسة منبع الصالحين المتوسطة

$$
\text { جرسيك. أما الدليل في هذا هو: }
$$

(أ) من نتائج الاختبار القبلي والاختبار البعدي للفصل التجريبي، أن هناك

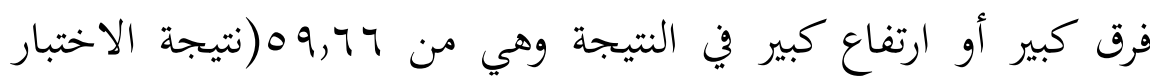

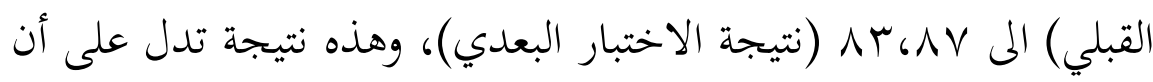

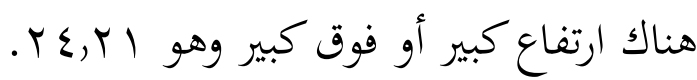
(ب) من نتيجة الإختبار حصلت الباحثة أن قيمة t-tes على فوق الحساب ونداب

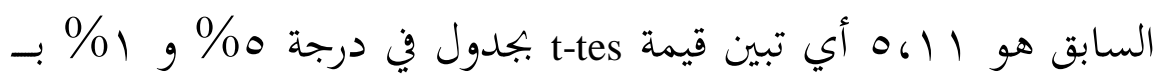




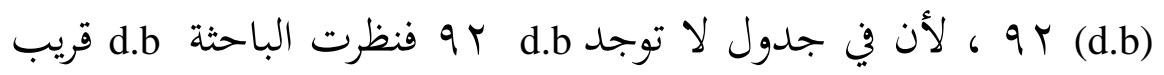

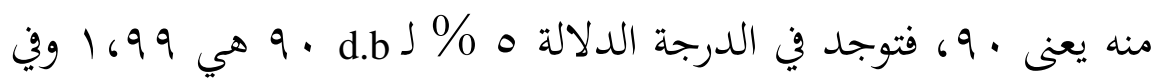

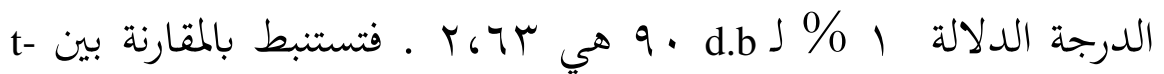

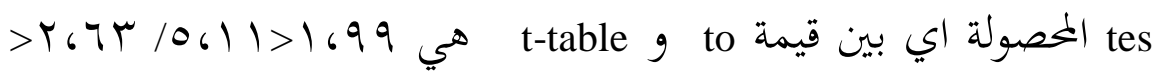

$$
0.11
$$

وخلاصة القول من المعلومات السابقة أن قيمة to او تاء الحسابي أكبر من قيمة t-table او تاء الجدول فالفرض الصفر (Ho) مردود، والفرض البدل (Ha) مقبول. هذا يشير الى أن المذكرة لشرح نظم العمريطي بخريطة المفاهيم تؤثر تأثيرا تفاعليا في ترقية فهم مادة النحو. س.قائمة المصادر والمراجع

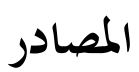

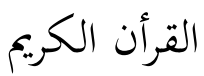

$$
\text { قاموس اللغة العربية (البشري) }
$$

أحد الطلاب من معهد لاعيتان ويداع توبان، نظم الجرومية وترجمته. العمرطي، شريف الدين يميى. نظم العمرطي على متن الأجرومية المراجع العربية - ماية

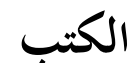

ابن جني، أبو الفتح عثمان. الخصائص، المجلل الأول، (بيروت : دار الكتاب العربي، دون التاريخ) 
أحمد طعيمة،رشدي. تعليم العربية لغير الناطقين بما مناهجه وأماليبه، ( الرياط: المنظمة

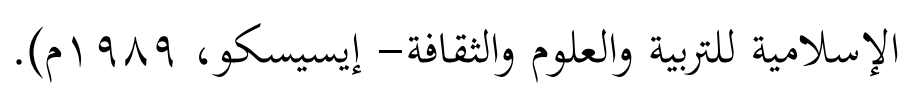

بحر الدين، أوريل. مهارات التدريس نحو إعداد مادر اللغة العربية الكفء، (مالانق :

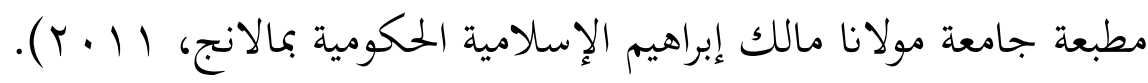

الخالي،محمد علي. الإختبار التحصيلية : إعدادها وإجراءها وتحليلها ( الأردن: دار

$$
\text { الفلاح، (1991) }
$$

دويدري، رجاء وحيد. البحث العلمي أساسياته النظرية وممارسته العملية، (دمشق : دار

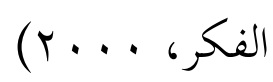

ديوي حميدة حنفي وأحمد محترام، قواعد النحو الميسرة، ( مالانق: مطبعة جامعة مولانا

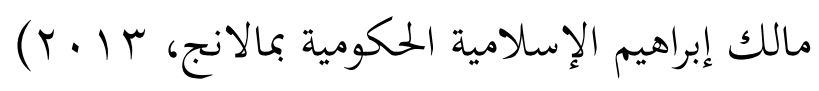

راتب قاسم عاشور ومحمد فؤاد الحوامدة، أساليب تلدريس اللغة العببية بين النظرية

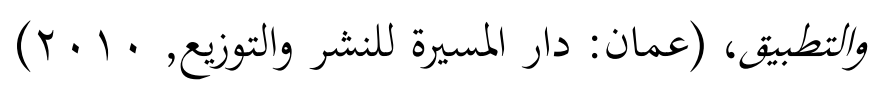

), الطناوي، عفت مصطفى. أساليب التعليم والتعلم وتطبيقاتما في البحوث التربوية

$$
\text { القاهرة: مكتبة الأنجلو المصرية، .... ب ). }
$$

عاطف الصيفي. المعلم واستراتيجيات التعليم الحلديث. (الأردن، عمان: دار أسامة للنشر

$$
\text { والتوزيع، } 9 \text {. . r. (r). }
$$

علي أحمد مدكور، رشدي أحمد طعيمة و إيمان أحمد هريدي، المرجع في مناهج تعليم

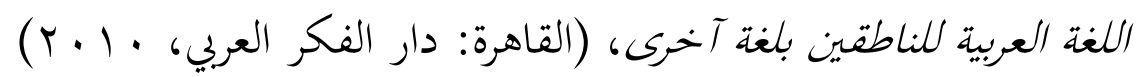


الغلاييني، مصطفى. V. V. T، جامع الدروس العربية، الطبعة الثامنة، بيروت : دار الكتب العلمية

القاسم، وجيه بن قاسم. ملخص لإستراتيجية التنديس بخرائط المغاهيم،( منشور في موقع مكتب التربية العربي لدول الخنليع).

محمود رشدي فاطر ومصطفى رسلان، تعليم اللغة العربية والتربية الدينية (القاهرة : دار

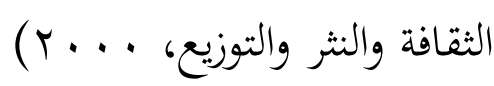

مدكور،علي أحمد. طرق تلديس اللغة العربية،( عمان: دار المسيرة للنشر والتوزيع، $(p r \cdot)$.

هادي،نور. الموجه لتعليم المهارات اللغوية لغير الناطقين بها، (مالانق: مطبعة جامعة

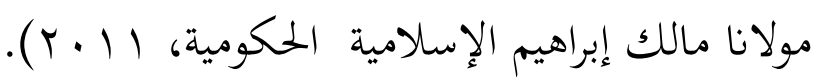

الهاشمي،أحمد. القواعد الأساسية للغة العربية، (القاهرة : طبعة مزيدة ومنقحة، ج . . T م) البحوث

ابن خليل. إعداد المواد النحوية باستخدام برنامج أدوب فلاش لتنمية مهارة القراءة

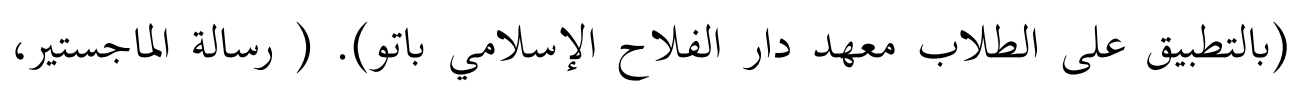

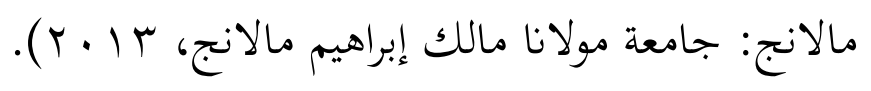

الدين، نصار. استخدام كتاب بتحيد النحو لشوقي ضيف لترقية تعليم النحو بالتطبيق

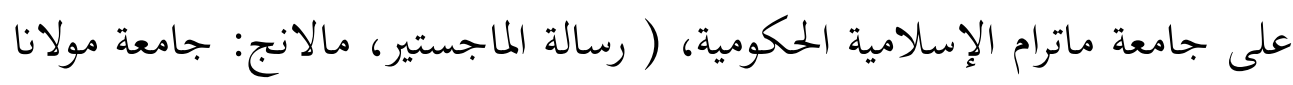

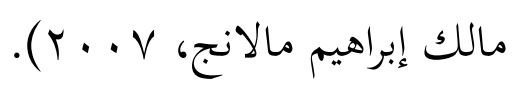


زهرة،ريني. " تطوير كتاب نحو الواضح باستخدام البرنامج Edraw Max "، ( رسالة

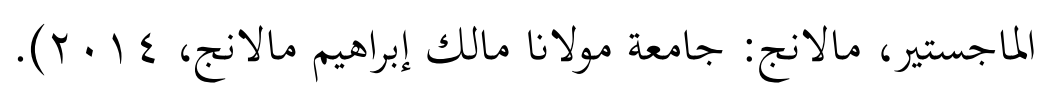

القيسي،بخيت. " أثر خرائط المفاهيمية في تحصيل طلبة المرحلة الأساسية وتفكيرهم الناقد

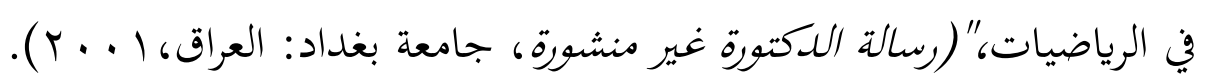

مبروري. استخدام الطريقة الإستقرائية والطريقة القياسية في تعليم قواعد اللغة العببية،(بال)

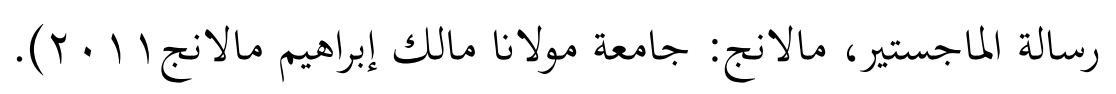

المطري،بشرة خميس هاشم."أثر التزود بالخرائط المفاهيمية على تحصيل الطلبة واتجاهاتم

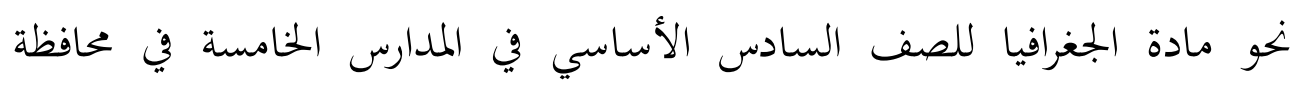
البلقاء" ( رسالة بجستير جامعة الشرق الأوسط للدراسات العليا كلية العلوم

$$
\text { الإنسانية قسم العلوم التربوية, و . . †). }
$$

النهي، عفة. تطوير المادة التعليمية لتعليم قواعد اللغة العربية،( رسالة الماجستير، مالانج:

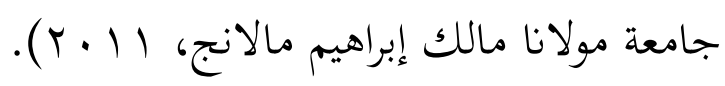

\section{الججلات والدوريات والنشرات}

أبو عقيل، إبراهيم. أثر استخدام الخرائط المفاهيمية في تدريس التفاضل وتنمية التفكير الإستدلالي لدى الطلبة الثانوية العامة ( الفرع العلمي) فلسطين، ( مجلة إتحاد إندان

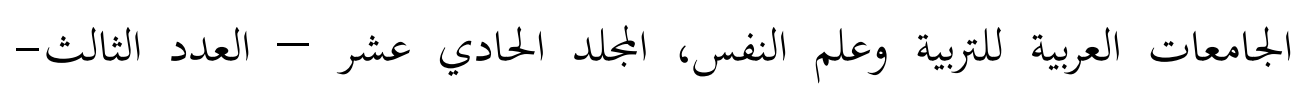
$\cdot(r \cdot 1 r$

السيد شهدة،السيد علي. "أثر استخدام خرائط المفاهيم في تدريس قوانين الغازات على قلق الطلاب وتصيلهم، الجمعية المصرية للمناهج وطرق التدريس،" (المؤتمر العلمي 


$$
\begin{aligned}
& \text { السادس، مناهج التعليم بين الايجابيات والسلبيات الإسماعيلية: م-1 ا أغسطس. } \\
& .(199 \varepsilon \\
& \text { عزواسماعيل عفانة، نائلة نجيب الخزندار، حسن ربحي مهدي، نصر خليل الكحلوت، } \\
& \text { طرق تلديس الحاسوب ( teaching computer method) } \\
& \text { نصر محمد خليفة مقابلة وغصايب محمد مطلق الفلاحات. أثر التدريس باستخدام } \\
& \text { الخرائط المفاهيمية على تحصيل طلبة الصف الثامن الأساسي لقواعد اللغة العربية } \\
& \text { في الأردن، مجلة جامعة دمشق المجلد بr العدد الرابع، • ( • . . }
\end{aligned}
$$
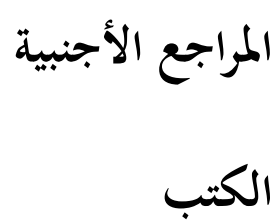

Abdul Hamid, Uril Baharuddin dan Bisri Musthofa. Pembelajaran Bahasa Arab pendekatan, metode, strategi, materi dan media,(Malang : UIN Malang Press, 2008)

Akbar, Sa'dun. Instrumen Perangkat Pembelajaran, ( Bandung, PT. Remaja Rosdakarya, 2005 )

Arifin, Zainal. Evaluasi Pembelajaran Prinsip Teknik Prosedur, ( Bandung, PT. Remaja Rosdakarya, 2005 )

Arikunto, Suharsimi. Prosedur Penelitian Suatu Pendekatan Praktek, (Rineke cipta : jakarta, 2006)

Bisri Mustofa dan Abdul Hamid. Metode dan Strategi pembelajaran Bahasa Arab,(Malang: UIN Maliki Press, 2011)

Buzan, Tony. Buku Pintar Mind Map ( Jakarta: PT Gramedia cetakan kesepuluh, 2012)

Musthofa, Syaiful. Stategi pembelajaran Bahasa Arab Inovatif, (Malang : UIN Maliki Press, 2011) 
Setyosari, Punaji. Metode Penelitian Pendidikan dan pengembangan, (Jakarta : Kencana, 2010)

Sugiyono, Metode Penelitian Kuantitatif, Kualitatif, dan $R \& D$, (Bandung: Alfabata,2008)

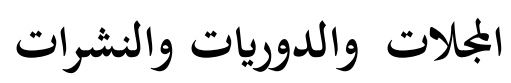

Alut, B. Concept Mapping A Study strategy in earth science Vol,15,( journal of College،1985).

Naidu, S. Concept mapping students workbook. Montreal, (Quebec: Confordia University,1990). (ERIC Document Reproduction Service No. ED 329 247).

Wandersee, J.n .Concept Mapping and the Cartography of cognition. Vol.27,No.10, (Journal of research in science teaching, 1990).

Zakiyah Arifa dan Dewi Hamidah. Pengembangan Bahan Ajar Qowaid Bahasa Arab berbasis Mind Map Untuk tingkat Perguruan Tinggi, Jurnal El Qudwah, ( Malang : LEMLITBANG UIN Malang, 2011)

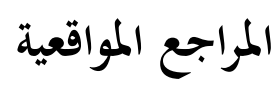

Arcana, Nyoman. https://www.scribd.com/doc/255125781/Panduan-

Menggunakan-Edraw-Mind-Map, diakses 11-01-2016 